\title{
INFORMACIÓN BIBLIOGRAFICA
}

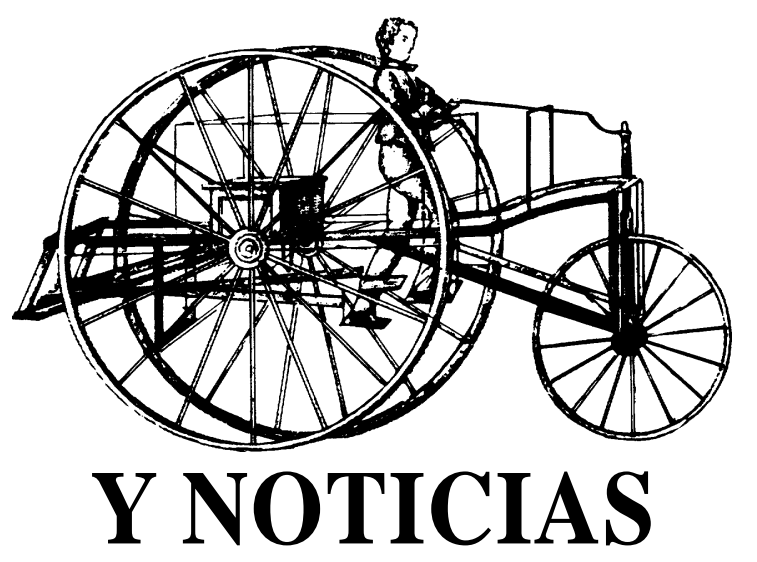

\section{TESIS DIDÁCTICAS*}

\begin{abstract}
* Recordamos que los datos que se precisan para la publicación de los resúmenes de tesis didácticas son los siguientes: título; autor o autora; tipo de tesis (doctoral o de maestría); director(es) o directora( s); departamento, universidad, programa en que se ha presentado; fecha de presentación; resumen de una extensión máxima de 4.500 caracteres, acompañado de disquete.
\end{abstract}

\begin{abstract}
OBSTACLES EN L'APRENENTATGE MATEMÀTIC: LA DIVERSITAT D'INTERPRETACIONS DE LA NORMA

Obstáculos en el aprendizaje matemático: La diversidad de interpretaciones de la norma

\section{Tesis doctoral}

Autora: Planas i Raig, Núria

Directora: Gorgorió i Solà, Núria

Lugar: Didàctica de la Matemàtica i de les Ciències Experimentals. Universitat Autònoma de Barcelona

Programa: Didàctica de la Matemàtica Fecha: 20 de julio de 2001

\section{Resumen}

Este trabajo de tesis doctoral se halla inscrito en el marco de los estudios que se ocupan del aprendizaje de las mate-

máticas desde una perspectiva sociocultural crítica (Lerman, 2000; Morgan, 2000; van Oers, 1996). Como en este tipo de estudios, se pretende poner de relieve la pluralidad de significados, valoraciones, legitimidades e identidades coexistentes en el aula de matemáticas y, a su vez, la gestión social de esta pluralidad.

Con ese propósito, se diseña una investigación cualitativa basada en un estudio de casos microetnográfico llevado a cabo en tres aulas de secundaria con jóvenes adolescentes en situación de riesgo social. Tal como muestra uno de los estudios piloto (Planas, 1999), el hecho de que las poblaciones de riesgo se encuentren especialmente afectadas por las interrupciones en la participación y presenten trayectorias de no-implicación muy marcadas ha de facilitar el acceso al conocimiento de factores comunicativos del aula de matemáticas que provocan obstáculos en el aprendizaje y que, en otras poblaciones, pueden estar quedando más encubiertos.
\end{abstract}

Los obstáculos en el aprendizaje matemático que se indagan son esencialmente de dos tipos: las dificultades comunicativas y los obstáculos comunicativos, definidos en una relación de interdependencia mutua. Por un lado, el término dificultad comunicativa se vincula a la naturaleza cultural del aula de matemáticas, en referencia a la ambigüedad que el alumno experimenta al intentar comprender significados usados en el aula diferentes a los inicialmente esperados. Por otro lado, el término obstáculo comunicativo se vincula a la naturaleza social del aula, en referencia a los impedimentos que el alumno encuentra en su entorno interpersonal al intentar resolver dificultades de comprensión de las prácticas en las que está implicado o bien al buscar espacios en los que se le permita comunicar significados personales y en los que se le comuniquen significados ajenos.

En el desarrollo de una aproximación interaccional, situamos los obstáculos mencionados en el contexto normativo 
del aula de matemáticas. La comprensión de las normas de actuación legitimadas en el aula y la adecuación del comportamiento y las formas de participación del alumno en función de estas normas son condiciones necesarias para acceder al discurso pedagógico principal. Sin embargo, las oportunidades de comprensión y de adecuación tienen que ver con la distancia cultural del alumno a los episodios del aula (dificultades comunicativas), su distancia social al resto de participantes (obstáculos comunicativos) y la particularidad de sus procesos individuales de dotación de sentido y de configuración de escenarios emocionales (experiencia de dificultades y obstáculos).

Dentro de este marco teórico general, se plantea la cuestión principal de la investigación: ¿Se pueden establecer conexiones entre la comprensión que un alumno tiene de las normas sociomatemáticas del aula y la aparición de obstáculos en su proceso de aprendizaje matemático? La interpretación de esta cuestión se realiza tratando de poner de manifiesto obstáculos en el aprendizaje matemático derivados de la complejidad sociocultural del aula de matemáticas Este objetivo se operativiza por medio de cinco objetivos específicos que marcan las sucesivas etapas de la fase empírica:

1) Identificar momentos de interrupción en los procesos individuales de aprendizaje matemático.

2) Identificar divergencias en la interpretación de las normas sociomatemáticas del aula y distancias culturales emergentes.

3) Identificar valoraciones asociadas al alumno y distancias sociales emergentes.

4) Identificar experiencias de distancia cultural y social.

5) Elaborar rutas explicativas para las interrupciones e indagar explicaciones en base a las distancias culturales, las sociales y la experiencia que el alumno tiene de ellas.

Tras haber analizado en profundidad veintidós casos de interrupciones en el aprendizaje matemático, algunas de las conclusiones más relevantes de la tesis son las siguientes:

a) Las trayectorias de participación matemática de alumnos inicialmente motivados por la tarea son muy frágiles. Los cambios de participación a no-participación, y al revés, son frecuentes y hacen que, en general, coexistan modelos, per- manentes o transitorios, de no-implicación con otros de implicación.

b) Los alumnos acostumbran a adoptar actitudes más trangresoras mientras participan en la tarea matemática que cuando se mantienen al margen. Casi siempre, configuran escenarios emocionales predominantemente positivos durante los intervalos de participación y, en cambio, otros negativos durante los intervalos de no-participación.

c) Aprender matemáticas y participar en el aula de matemáticas significa, también y necesariamente, aprender las normas sociomatemáticas que la regulan. Los alumnos que no dominan estas normas pueden ver seriamente cuestionada su participación y, como consecuencia, pueden ver reducidas sus oportunidades de aprendizaje.

d) Aprender y aplicar normas legitimadas no es fácil. Existe una gran tensión entre los procesos enculturadores del aula de matemáticas, que pretenden comunicar normas canónicas, y los procesos de deslegitimación, que pretenden aislar los emisores de significados minoritarios, en especial los relativos a normas de la práctica matemática.

e) La alta frecuencia de obstáculos comunicativos que agravan las dificultades comunicativas con las que se encuentran algunos alumnos al intentar comprender las normas de actuación legitimadas muestra que la dimensión social del aula de matemáticas es una auténtica fuente problematizadora de la diversidad cultural.

En definitiva, encontramos que la experiencia de distancia cultural y social, vivida por el alumno al percibir significados que le son ajenos y valoraciones negativas, está íntimamente relacionada con la experiencia de dificultades comunicativas y obstáculos para resolver estas dificultades. La vivencia reiterada de distancias culturales y sociales influencia las formas de participación en el aula, y la asignación de escenarios emocionales negativos a esta vivencia puede comportar múltiples interrupciones en las trayectorias globales de implicación y generar perfiles permanentes de no-participación con los correspondientes historiales crónicos de fracaso escolar y de frustración personal.

\section{Referencias bibliográficas}

Lerman, S. (2000). The social turn in the mathematics education research, en Boaler, J. (ed.). Multiple perspectives on mathematics teaching and learning, pp. 19-44. Wesport: Ablex-Publ.
Morgan, C. (2000). Discourses of assessment-discourses of mathematics, en Matos, J.P. y Santos, M. (eds.). Proceedings of the $2^{\text {nd }}$ International Mathematics Education and Society Conference, pp. 58-76. Montechoro. Portugal.

Planas, N. (1999). Ambient de resolució de problemes en una classe multiètnica: identificació de norma social, norma sociomatemàtica i norma matemàtica. Tesis de maestría. Departament de Didàctica de la Matemàtica i de les Ciències Experimentals. Universitat Autònoma de Barcelona.

Van Oers, B. (1996). Learning mathematics as a meaningful activity, en Steffe, L.P. et al. (eds.). Theories of mathematical learning, pp. 91-114. Hillsdale: Lawrence Erlbaum Ass. Publ.

\section{LA INTERVENCIÓN DE LA MEMO- RIA DE TRABAJO EN EL APREN- DIZAJE DEL CÁLCULO ARITME- TICO}

\section{Tesis doctoral}

Autor: Alsina i Pastells, Àngel Directora: Sáiz Roca, Dolors Departamento: Psicologia de l'Educació. Universitat Autònoma de Barcelona Fecha: 7 de mayo de 2001

Esta tesis doctoral analiza la intervención de la memoria de trabajo en el cálculo. Con ello, se pretende responder a algunas cuestiones básicas relativas a los procesos de aprendizaje del cálculo y las causas que inciden en la aparición de dificultades. Para alcanzar este objetivo, la tesis consta de un apartado teórico en el que se plantea, en primer lugar, una breve revisión de las principales teorías psicológicas que han incidido en el aprendizaje del cálculo; en segundo lugar, se revisan los trabajos sobre dificultades de aprendizaje del cálculo; y, por último, un tercer capítulo aborda la problemática de los estudios sobre memoria de trabajo y cálculo: a) gran diversidad metodológica en las pruebas usadas, en el control de la validez y fiabilidad, en el tipo de diseño, etc.; $b$ ) inexistencia de trabajos al iniciar el estudio que verifiquen en los mismos sujetos la intervención conjunta 
de los tres subsistemas de la memoria de trabajo; y $c$ ) inexistencia también de trabajos que hayan diseñado un programa de activación específico para la optimización y potenciación de la memoria de trabajo.

El apartado empírico presenta la investigación realizada en dos fases para verificar los siguientes objetivos:

a) la intervención global de la memoria de trabajo en el cálculo;

b) la intervención específica de los distintos subsistemas de la memoria de trabajo (bucle fonológico, agenda visoespacial y ejecutivo central) en el cálculo;

c) el efecto de un programa de activación de la memoria de trabajo en la capacidad de memoria de trabajo y en el cálculo.

La primera fase consiste en administrar, a una muestra de 94 niños de 7-8 años escolarizados en cinco colegios ubicados en la Cataluña central, diferentes pruebas de medida del rendimiento académico en numeración y cálculo, elaboradas de acuerdo con el currículo de matemáticas de primaria del Departament d'Ensenyament de la Generalitat de Catalunya (1992), así como distintas pruebas de la «Bateria de tests de memòria de treball» de Pickering, Baqués y Gathercole (1999). Los resultados obtenidos son los siguientes:

- Los niños con peores recursos de memoria de trabajo son los que rinden menos en tareas de numeración y cálculo; los que tienen más recursos de memoria de trabajo son los que obtienen mejores rendimientos; y los que tienen un nive medio de memoria de trabajo obtienen también niveles de rendimiento intermedio en tareas de numeración y cálculo.

- La tendencia se repite tanto al considerar las tareas de numeración y cálculo globalmente como por separado.

- La relación se confirma tanto al estudiar el rendimiento de la memoria de trabajo en función del nivel de numeración y cálculo (bajo, medio, alto) como a la inversa, es decir, al estudiar el rendimiento en tareas de numeración y cálculo en función del nivel de memoria de trabajo (bajo, medio, alto).

- En nuestro trabajo se establece, por primera vez, una relación entre memoria de trabajo y cálculo en escolares españoles. La tendencia de los escolares españoles es similar a la de otros niños de culturas occidentales.
- Tanto al considerar las tareas de numeración y cálculo globalmente como por separado, se produce una relación estadísticamente significativa con los recursos de dos de los tres subsistemas de la memoria de trabajo: bucle fonológico y ejecutivo central, aunque la relación más consistente se da con el ejecutivo central. Respecto a la agenda «visoespacial» los resultados obtenidos indican una escasa incidencia en tareas de numeración y cálculo.

- La relación se confirma tanto al estudiar el rendimiento de los distintos subsistemas de la memoria de trabajo en función del nivel de numeración y cálculo como a la inversa, es decir, al estudiar el rendimiento en tareas de numeración y cálculo en función del nivel de los distintos subsistemas de memoria de trabajo.

En la segunda fase se parte de una muestra de 50 niños (25 en el grupo experimental y 25 en el grupo control) que han formado parte ya de la muestra de la primera fase. Los dos grupos, antes de iniciar la segunda fase, no presentan diferencias estadísticamente significativas en ninguna de las pruebas administradas en la primera fase. Al iniciar la segunda fase, los sujetos del grupo experimental reciben la aplicación de un programa de activación de la memoria de trabajo diseñado para esta tesis. El programa consta de 40 sesiones de aproximadamente 45 minutos cada una, y se aplica durante dos trimestres escolares. Al final de esta aplicación, se recogen nuevos datos empíricos con el objeto de contrastar los resultados respecto al grupo control y, de esta forma, determinar la posible incidencia del programa. Los principales resultados obtenidos son:

- Todos los niños de 7-8 años de nuestra muestra (grupo experimental y control) tienden a incrementar su rendimiento en tareas de memoria de trabajo.

- El programa de activación de la memoria de trabajo ejerce un claro efecto en el rendimiento del bucle fonológico y sobretodo del ejecutivo central, puesto que los sujetos del grupo experimental obtienen incrementos estadísticamente superiores respecto al grupo control.

- El programa ha conseguido también mejorar el rendimiento en pruebas de la agenda «viso-espacial», aunque los incrementos son inferiores.

- El programa se muestra efectivo sobre todo en niños que parten de un nivel más bajo de memoria de trabajo.

- Los análisis cualitativos realizados confirman que prácticamente todos los niños del grupo experimental tienden a aumentar las puntuaciones en todas las pruebas de memoria de trabajo, mientras que los niños del grupo control, aunque no se puede generalizar, tienden a mantener o incluso a disminuir las puntuaciones.

- Todos los niños de 7-8 años de nuestra muestra tienden a aumentar sus puntuaciones en tareas de numeración y cálculo, lo cual es absolutamente lógico; si no, debería cuestionarse la incidencia de la labor educativa.

-El programa ejerce un claro efecto en el rendimiento en tareas de numeración y cálculo, ya que los niños del grupo experimental obtienen incrementos estadísticamente superiores respecto al grupo control.

De los resultados se desprenden distintos objetivos didácticos, de entre los que destaca el hecho de que el tratamiento de los trastornos de cálculo no debe basarse exclusivamente en la repetición y la práctica, sino en la potenciación de capacidades psicológicas básicas como la memoria de trabajo.

INFORMATIZACIÓN DE UNA ASIGNATURA. DESARROLLO DEL ENTORNO GAME Y APLICACIÓN A LA TERMODINÁMICA TÉCNICA

\section{Tesis doctoral}

Autora: Velasco Callau, M. Carmen Directores: Martínez, Begoña y Turégano, José Antonio

Lugar: Departamento de Didáctica de las Ciencias Experimentales de la Universidad de Zaragoza

Programa: Didáctica de las Ciencias Experimentales

Fecha: 12 de febrero de 2001

\section{Marco teórico}

Su carácter es marcadamente pluridisciplinar, ya que confluyen la didáctica, la informática y los aspectos científicotecnológicos representados en este caso por la termodinámica técnica.

Se pone en combinación un conjunto de elementos de cada uno de dichos campos 
y sus implicaciones docentes, en el marco de lo que viene llamándose la sociedad de la información, para proponer un modelo didáctico de programación de una asignatura con el fin de que aproveche todo el potencial de los nuevos recursos tecnológicos para optimizar los esfuerzos y los resultados de los implicados en la docencia y sus procesos de enseñanza y aprendizaje.

Se analiza el contexto actual tecnológico en la sociedad de la información y sus implicaciones en el sistema educativo; se revisan los diversos enfoques de la enseñanza y el fundamento psicológico de las teorías del aprendizaje, estableciendo la relación entre la enseñanza informatizada y dichas teorías. Se destaca la necesidad de modificar la tradición verbalista de la enseñanza a la vista de las nuevas necesidades de nuestros estudiantes y el tipo de preparación que está demandando la nueva sociedad, lo que requiere combinar el conocimiento psicológico con los nuevos medios tecnológicos, para propiciar que:

- el estudiantado adquiera el protagonismo de su propio aprendizaje ( aprendizaje significativo);

- el profesorado asuma el papel de coordinador-orientador de dicha tarea.

Este aprendizaje significativo se desarrolla de manera óptima dentro del modelo constructivista. Se pretende que la incorporación de los nuevos medios tecnológicos facilite el cambio metodológico requerido por el mismo, mediante el desarrollo de materiales informáticos que, en combinación con las tecnologías de la información y la comunicación (TIC), promuevan el autoaprendizaje de los estudiantes y el papel de orientadoranimador del profesorado.

\section{Aportaciones}

Se desarrolla un modelo de entorno abierto de enseñanza-aprendizaje: GAME (sistema autor-gestor hipermedia), en el que la combinación de métodos y medios proporciona un conjunto organizado de recursos y un sistema de gestión del mismo para facilitar tanto a los estudiantes como a los profesores el cambio metodológico, mediante la aplicación de una metodología didáctica basada en las relaciones conceptuales, las cuales son el soporte del sistema organizativo del conjunto.

Esto se aplica a la asignatura de Termodinámica Técnica, dotándola de material multimedia de autoaprendizaje y herramientas didácticas para la resolución de problemas y cuestiones.

\section{Metodología}

Se plantea un paralelismo entre la programación de una asignatura convencional y dicha asignatura informatizada, para establecer los materiales más convenientes a desarrollar. Se analizan las distintas etapas de planificación y organización de un curso, estudiando las nuevas necesidades que la informatización plantea en sus diversos aspectos y donde los diferentes métodos de enseñanza tradicionales y las actividades tienen su transposición en el modelo informatizado conformando el entorno GAME que se completa con el desarrollo del sistema gestor-autor, el cual:

- En modo «Usuario» gestiona el conjunto de los diferentes componentes del sistema. Es como lo manejan los estudiantes en su trabajo personal o los profesores en clase.

- Gestiona las comunicaciones sobre la red en acciones como tutorías y evaluación.

- Mantiene una base de datos interna con información de usuarios, condiciones de acceso...

- En modo «Autor» facilita al profesorado la creación, a partir de la base de datos general, de los diferentes materiales informáticos: clases unimedia, hipertexto, prácticas de simulación...

El modo «Gestor» incluye los siguientes elementos:

- El hipertexto: Está presentado a través de la pantalla principal. Son los contenidos temáticos de la asignatura similar a un libro electrónico con diferentes procedimientos de acceso a la información, pero dotado de una estructura mucho más potente. Al conectarse con la organización conceptual, y por el sistema de búsquedas y conexiones con el que relaciona todos los materiales y herramientas disponibles sirve de base para toda la estructura general del entorno «game».

- El glosario: Es una lista de conceptos con un sistema de búsqueda definida por el profesor/a -en función de sus criterios didácticos-y para evitar pérdidas o desorientaciones en el espacio hipertextual, y un primer nivel de conexiones, o nivel elemental, dirigido a los estudiantes novatos.

- La pantalla de conceptos y relaciones: Contiene las relaciones y las conexiones de los conceptos con sus relacionados y con los elementos o materiales auxiliares, como pueden ser ejercicios, cuestiones, exámenes, etc. Las conexiones aquí corresponden al nivel superior o de ex- perto, las cuales permiten llevar a cabo un estudio o un repaso más estructurado de carácter constructivista, mediante la relación entre conceptos y la jerarquización de los mismos, y el uso de mapas conceptuales que ayuden a completar la propia estructuración del conocimiento.

- Materiales auxiliares: Materiales de carácter algorítmico, se cargan en el programa mediante un motor, y materiales que mantienen su estructura independiente: el gestor actúa simplemente de lanzador del recurso, como las prácticas de simulación, las unidades multimedia, las herramientas...

Se utilizan dos elementos adicionales: la base de datos que contiene los materiales digitalizados y las relaciones entre los elementos de la estructura, y el visor, incorporado para agilizar la visualización de dichos materiales. Además, para el entorno de enseñanza, el asistente permite que el profesor/a pueda preparar su sesión de instrucción previamente, usando la misma estructura de conexiones que los estudiantes van a tener después para su autoaprendizaje.

Los principales medios desarrollados para el modelo informatizado en termodinámica técnica son:

- Materiales unimedia: Desarrollos informáticos tipo multimedia, para ser usados por el profesorado en las sesiones expositivas.

- Materiales multimedia: Abarcan las explicaciones teóricas y conceptuales de la asignatura, y están dotados de los elementos multimedia (imágenes, animaciones, vídeos, etc.) y de la interactividad necesaria para que los estudiantes puedan utilizarlos en autoaprendizaje.

- Materiales de simulación: Para uso en laboratorio virtual además de las prácticas reales.

- Herramientas específicas: Son programas que proporcionan un entorno instrumental específico de cada materia. Permiten la realización de tareas procedimentales, cálculo, representaciones gráficas, análisis de conceptos, etc. Para la termodinámica técnica se han desarrollado: Termograf (análisis de conceptos y representaciones gráficas), ARIES (resolución de problemas) y PropSust (cálculo de propiedades y el análisis de procesos).

- Programas para evaluación: Evaluación formativa en clase para el profesor/a y autoevaluación para los estudiantes y la evaluación sumativa, que puede realizarse bajo demanda, y utilizando la red. 


\section{Conclusiones}

La dualidad unidad temática - conceptos para la presentación de los contenidos y la estructura de conexiones entre los elementos disponibles le dotan de su elevado potencial didáctico. El análisis y la categorización de los conceptos y su estructuración mediante mapas y relaciones conceptuales son una potente herramienta para facilitar el aprendizaje significativo de los estudiantes. La herramienta informática creada es un recurso fácil de explotar y con grandes posibilidades.

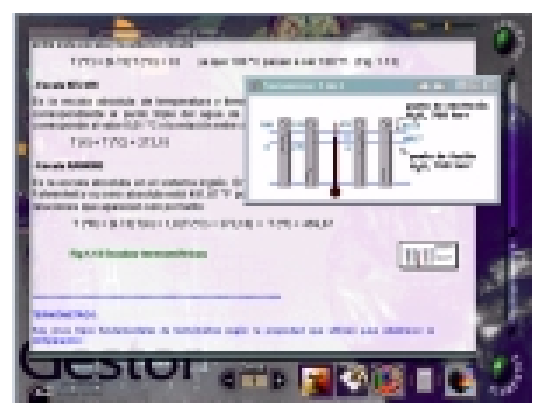

Pantalla principal del gestor

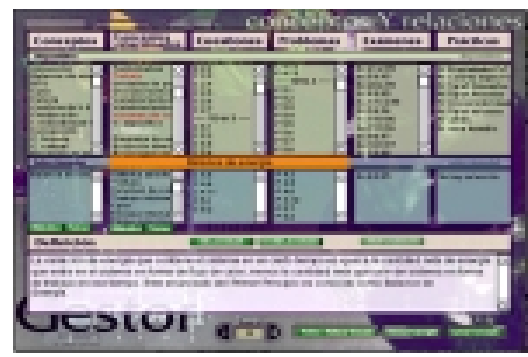

Pantalla de relaciones conceptos y materiales
CONSTRUYENDO SIGNIFICADOS EN MECÁNICA CUÁNTICA: RESULTADOS DE UNA PROPUESTA DIDÁCTICA APLICADA A ESTUDIANTES DE FISICA GENERAL

Tesis doctoral

Autora: Greca, Ileana María

Directores: Moreira, Marco Antonio y

Elnecave Herscovitz, Victoria

Lugar: Instituto de Física. Universidade Federal do Río Grande do Sul. Porto Alegre. Brasil

Fecha: 2 de octubre de 2000

La importancia creciente de la mecánica cuántica (MC) en la tecnología hace ineludible que se propicie a los estudiantes de cursos universitarios de ciencias exactas conocimientos básicos de MC que les permitan profundizar en el estudio del mundo microscópico y los incentive para ello. Los resultados de varias investigaciones muestran que esto es difícilmente obtenido en las disciplinas tradicionales introductorias de MC: los estudiantes trabajan básicamente manipulando fórmulas y su entendimiento de los conceptos cuánticos es superficial. Sin embargo, son escasas las investigaciones sobre estrategias didácticas que faciliten su aprendizaje.

Nos propusimos investigar las dificultades de estudiantes universitarios en relación con los conceptos cuánticos fundamentales y elaborar e implementar una estrategia didáctica que facilitase su comprensión. La base teórica adoptada es la teoría de los modelos mentales de Johnson-Laird, según la cual la adquisición de conceptos está mediada por la construcción de modelos mentales de los mismos. Tales modelos se construyen a partir de ciertos núcleos que determinarían la forma de visualización de fenómenos o situaciones. Así, las dificultades que los estudiantes encontrarían para comprender conceptos cuánticos fundamentales derivarían de la necesidad de abandonar formas de visualización clásicas del mundo microscópico, implicando un cambio en los núcleos a partir de los que construyen sus modelos mentales. En particular, consideramos que las ideas subyacentes a los conceptos de superposición lineal de estados, principio de incertidumbre (dualidad ondapartícula) y carácter probabilístico de los resultados de medida deberían estar en los nuevos núcleos para la comprensión de la MC.

En la primera etapa, investigamos diferentes grupos de estudiantes universitarios que recibieron instrucción tradicional en contenidos introductorios de MC. Partiendo de la premisa de que si dos conceptos o situaciones se perciben como semejantes es porque los modelos mentales generados para comprenderlos contienen elementos comunes, estando esos elementos comunes en los núcleos de los mismos, construimos un instrumento (test de asociación de conceptos y problemas conceptuales) aplicado antes y después de la instrucción. También utilizamos entrevistas como fuentes de información. Los datos fueron analizados de forma integradora, usando análisis interpretativo, de agrupamientos jerárquicos y multidimensional.

En estudiantes de Física General IV de ingeniería encontramos los núcleos de partícula clásica, sintético y electrón difuso, variaciones de formas de visualización clásicas, que impiden la comprensión adecuada de conceptos cuánticos fundamentales. Para muchos estudiantes no fue posible identificar ningún núcleo, reflejado en el uso mecánico de definiciones y fórmulas inconexas. En una disciplina más avanzada de la licenciatura en física, encontramos los núcleos ondulatorio y formal. El primero sigue siendo una visualización clásica de conceptos cuánticos y sólo el último, encontrado en un único estudiante, permite una comprensión adecuada de los conceptos cuánticos.

Evaluamos, entonces, que sería necesario un enfoque didáctico que facilitase el cambio de visualización clásica, argumentando a favor de una estrategia de cambio perceptual denominada de fenomenológica-conceptual (fenomenológica para propiciar la creación de una nueva percepción y conceptual, pues los fenómenos escogidos deben mostrar claramente la esencia de los conceptos implicados), destacando las características cuánticas de los sistemas en vez de la búsqueda de analogías clásicas. Así, utilizamos resultados recientes del área de fundamentos de MC, que evidencian estos conceptos y pasan a los estudiantes un aire de actualidad sobre la MC. Esta estrategia fue implementada en tres grupos de estudiantes de Física General IV de ingeniería durante los dos semestres de 1999.

La evaluación de la propuesta (con instrumentos semejantes a los descriptos y resulados convergentes en los distintos análisis) nos permitió inferir que, a diferencia de lo que ocurría con el enfoque tradicional, una parte significativa de los estudiantes (alrededor de 65\%) conseguía construir núcleos que denominamos de cuántico completo e incipiente. que permitían «lecturas» cuánticas de fenómenos del mundo microscópico para ese nivel de instrucción. Los estudiantes 
que parecen no construir estos núcleos intentarían, como en el caso de la enseñanza tradicional, significar los conceptos cuánticos a partir de núcleos derivados de la fenomenología clásica o trabajar con fórmulas aisladas. La superposición lineal de estados parece ser el concepto de más difícil comprensión. Los estudiantes de los grupos experimentales obtuvieron en los problemas medias superiores en relación con otros dos grupos tomados de control.

La propuesta didáctica implementada no sólo pareció ser exitosa en la promoción de un cambio perceptual en una parte significativa de los estudiantes, sino que consiguió que muchos de ellos se quedasen extremadamente interesados en el asunto, siendo muy bien recibida por parte de los sujetos implicados (estudiantes y docentes), como muestran los resultados de los análisis realizados sobre esta cuestión.

\section{TRANSPOSICIÓN DIDÁCTICA DE LA ECUACIÓN DE CLAUSIUS- CLAPEYRON}

Del «saber sabio» al «saber enseñado en un laboratorio de escuela de ingeniería técnica»

\section{Tesis doctoral}

Autora: Torrecilla Daniel, María Isabel Director: Arlegui de Pablo, Javier

Lugar: Departamento de Didáctica de las Ciencias Experimentales. Universidad de Zaragoza

Programa: Didáctica de las Ciencias Experimentales

Fecha: 5 de junio de 2000

La tesis realiza el estudio del proceso transpositivo de la ecuación de ClausiusClapeyron. Dicho estudio integra el análisis del saber en tres estadios («saber sabio», "saber a enseñar en libros universitarios de texto», «saber enseñado en situación de prácticas de laboratorio») y el análisis de tres contextos de transición (científico-técnico previo, institucional, y contexto próximo).

El estudio del contexto científicotécnico previo muestra el estado de la ciencia sobre el calor a principios del siglo XIX. Para estudiar la génesis y desarrollo del saber sabio, se han estudiado (con un esquema común) artículos originales de Carnot, Clapeyron, Thomson, Clausius y Gibbs y se ha enmarcado el saber en el contexto científico actual. El análisis destaca el papel esencial jugado por la ECC en el establecimiento de la termodinámica, el carácter dual del campo problemático ligado a su origen (técnico y teórico) y el variado papel que tiene la ecuación en la compleja interrelación entre los campos problemático, teórico y experimental.

El análisis de los libros de texto muestra aspectos transpositivos generales e incide en aquéllos relacionados con el saber Ecuación de Clausius Clapeyron (alto nivel formal del sistema deductivo; insistencia en su carácter de herramienta de cálculo; triple desintetización en los manuales de prácticas)

El análisis transpositivo hasta la puerta del laboratorio enlaza con el realizado dentro de él mediante un cuestionario (analizador) que permite: $a$ ) caracterizar el saber enseñado en relación con la concepción previa y explícita que nos forjamos del mismo; $b$ ) comparar este estado del saber con los estadios anteriores; $y c$ ) detectar obstáculos didácticos.

Además de las relaciones didácticas, el analizador considera la integración de dos prácticas sociales de referencia (la científica y la del ingeniero técnico), que genera un modelo sistémico de laboratorio, y la concepción del proceso de aprendizaje en termodinámica como un proceso de modelización. El cuestionario se aplica a la transcripción de seis filmaciones de una práctica vinculada a la ecuación de Clausius-Clapeyron.

La tesis expone una serie de variables generales a considerar en el sistema didáctico y propone algunos cambios en su valor actual. Hace también propuestas concretas para que la actividad de laboratorio, enmarcada en el doble eje científico y técnico y a la luz del desarrollo histórico, posibilite una mayor interrelación entre los campos teórico, problemático y empírico, y una mayor sintetización de los saberes parciales que constituyen el saber.
MODELOS MENTALES DE CÉLULA: UNA APROXIMACIÓN A SU TIPIFICACIÓN CONESTUDIANTES DE COU

\section{Tesis doctoral}

Autora: Rodríguez Palmero, M. Luz Directores: Moreira, Marco Antonio y Marrero Acosta, Javier

Lugar: Departamento de Didáctica e Investigación Educativa y del Comportamiento. Centro Superior de Educación.

Universidad de La Laguna

Programa: Desarrollo del currículo y cambio en educación

Fecha: 2 de junio de 2000

El objeto de la investigación ha sido explorar el alcance y el grado de aplicación de la teoría de los modelos mentales de Johnson-Laird en el estudio de las representaciones generadas por el alumnado de COU con respecto al contenido celular trabajado en el aula. Partiendo de este referente teórico, se han procurado identificar y tipificar los modelos mentales de célula construidos por los estudiantes, así como su evolución a lo largo de un curso escolar, usando para ello sus producciones y verbalizaciones como representaciones externas o formas de plasmar ese conocimiento. El problema fundamental al que nos enfrentamos es:

- ¿Qué modelos mentales generan los estudiantes de COU cuando aprenden la célula?

Los objetivos propuestos son:

- Explorar el alcance y grado de aplicación de la teoría de los modelos mentales de Johnson-Laird en los procesos de aprendizaje de estudiantes de COU relativos a la célula.

- Identificar y tipificar los modelos mentales de célula definidos desde esta perspectiva en dichos estudiantes a partir de la interpretación de sus producciones y verbalizaciones.

- Delimitar los grados de evolución o cambio de las representaciones construidas por estos jóvenes a lo largo de un curso escolar completo.

- Elaborar un esquema global de análisis de esas producciones y verbalizaciones para representar la estructura y el funcionamiento celular.

El proceso de indagación seguido se articula en torno a: $a$ ) una primera tarea de revisión de lo que la investigación edu- 
cativa aporta con respecto al tema; $b$ ) una revisión bibliográfica relativa a la consideración de modelos mentales como formas de estudiar las representaciones; $c$ ) una exposición de la teoría que actúa como marco teórico de referencia en este proceso; $d$ ) la presentación de los aspectos metodológicos (estudio de casos) y las fases de la indagación; y, por último $e$ ) la discusión de los hallazgos y resultados, amén de las referencias bibliográficas utilizadas. Se incluyen treinta y nueve anexos que contemplan: análisis del contenido, contexto pedagógico, fuentes de recogida de información, contrastación y treinta y cinco informes de interpretación de los estudiantes considerados (el informe restante se ha incluido como ejemplo en el cap. 3).

El contexto de la investigación se corresponde con las condiciones naturales de aula en la asignatura de Biología de COU. La profesora titular ha actuado como investigadora, cuyo diario de sesiones se anexa. El análisis e interpretación de los datos se ha llevado a cabo en fecha posterior a la finalización del curso. Los casos indagados son los 36 estudiantes que han cursado oficialmente la asignatura, cuyos informes individualizados figuran en los anexos correspondientes. Como el objeto es interpretar lo que estos jóvenes hacen y dicen en los diferentes contextos escolares y de aprendizaje, se trata de un análisis del nivel psicológico de la cognición, y por ello se ha entendido que estos estudiantes debían estar en las condiciones más naturales posibles. Se ha considerado que el alumnado en cuestión en ningún momento debía saber que estaba siendo objeto-sujeto de investigación, de manera que no hubiera influencia alguna sobre su modo de pensar que no fuera lo normal en las aulas de Biología. Además, se ha optado por cuidar este aspecto porque se entiende que deben buscarse mecanismos de indagación que sean aplicables a estas condiciones naturales de aula y que puedan salir de ellas, así como que se puedan utilizar en las mismas porque de lo que se trata es de generar conocimiento sobre esos procesos cognitivos que son los que normalmente se producen y no los que se desarrollan en condiciones controladas de laboratorio. Esto es lo que justifica, no sólo la ignorancia de su papel esencial como protagonistas de investigación de estas 36 personas, ya que desconocían esta condición, sino también que todos y cada uno de ellos se hayan tenido en cuenta, ya que son los que lo cursaron en su totalidad. Las fuentes utilizadas para la recogida de información están constituidas por las producciones y verbalizaciones hechas por el alumnado a lo largo del curso escolar. Estos registros son los siguientes: los cuestionarios inicial y final (el mismo en ambas ocasiones); los cinco exámenes elaborados a lo largo del curso; los tres mapas conceptuales solicitados también en momentos diferentes, la interpretación de un símil de la célula, la elaboración de un dibujo relativo a su estructura y funcionamiento y la entrevista final. Se han cumplimentado individualmente en las horas ordinarias de clase (excepto la entrevista que se desarrolló una vez acabado el curso escolar) y los únicos que han sido susceptibles de calificación son los exámenes, extremo este que el alumnado conocía.

Se concluye, del trabajo desarrollado, que:

- El alumnado, para la interpretación que realiza de la célula y de su significado biológico, elabora modelos mentales que son identificables a través de sus producciones verbales, escritas y gráficas.

- Los modelos mentales relativos a la célula construidos por los estudiantes son susceptibles de tipificación y clasificación. Los que se han hallado son: modelo mental A o estructural, modelo mental B o dual, modelo mental C o causal discursivo, modelo mental D o causal «imagístico».

- Esos modelos mentales evolucionan al procesar la nueva información para reinterpretarla cuando se expone al alumnado a un proceso sistemático de construcción del conocimiento.

- En la construcción de modelos mentales explicativos de célula es necesario construir significativamente conceptos de elementos, propiedades y características y relaciones e interacciones entre ellos.

En lo que se refiere al planteamiento metodológico, el esquema de análisis propuesto se ha mostrado eficaz al especificar los niveles (selección, uso, imágenes), los criterios contemplados (frases, calidad del discurso, uso de la información, interpretación, deducción, jerarquización, significatividad, recurso analógico) y las categorías dentro de los mismos, permitiendo con ello obtener las interpretaciones que sobre las representaciones mentales entendidas como modelos han generado los estudiantes, así como su evolución a lo largo de un curso escolar.

A juzgar por los hallazgos y resultados obtenidos, se puede admitir que:

- La teoría de los modelos mentales de Johnson-Laird ofrece explicaciones y predicciones comprensibles, plausibles y fructíferas para el estudio de las representaciones mentales generadas por el alumnado relativas a la célula.
- Parece confirmarse la necesidad de abordar el aprendizaje de forma gradual y por aproximaciones sucesivas que facilite la incorporación y la interacción de los elementos conceptuales correspondientes, fundamentalmente cuando se trata de conceptos altamente estructurados, como la célula para este nivel de enseñanza.

- Se propone una sistematización para analizar las producciones y verbalizaciones de los estudiantes que facilite su interpretación.

- Se constata que el diagnóstico de representaciones mentales tiene que ser continuado y procesual para que tenga eficacia.

La investigación ha supuesto, por tanto, comprensión sobre lo que ocurre en las mentes de los estudiantes cuando se enfrentan a la célula como contenido, ya que ha aportado explicaciones y predicciones sobre sus modos de pensar al respecto, explicaciones y predicciones (en términos de Johnson-Laird) -modelos mentales del investigador- que, lógicamente, tienen consecuencias pedagógicas, lo que ha quedado claramente de manifiesto en las producciones y las verbalizaciones del alumnado, así como en su interpretación.

RAZONAMIENTOCOMBINATORIO EN ESTUDIANTES CON PREPARACIÓN MATEMÁTICA AVANZADA

\section{Tesis doctoral}

Autor: Roa Guzmán, Rafael

Directores: Díaz Godino, Juan y Batanero Bernabeu, Carmen

Lugar: Facultad de Educación. Departamento de Didáctica de la Matemática. Universidad de Granada

Programa: Didáctica de la Matemática Fecha: Mayo de 2000

En esta investigación se analizan las estrategias de resolución de una muestra de problemas combinatorios elementales, así como las dificultades y los errores encontrados, en estudiantes de los últimos cursos de la licenciatura de matemáticas. 
El estudio se realiza mediante cuestionarios escritos aplicados, en tres fases, a un total de 147 estudiantes y entrevistas individuales a una muestra reducida. La caracterización de los conocimientos puestos en juego por los estudiantes se ha realizado mediante el análisis de las respuestas a los cuestionarios y entrevistas, usando métodos cuantitativos y cualitativos.

Se ha estudiado la dificultad que presentan estos problemas combinatorios para estudiantes con una fuerte preparación matemática y se han comparado los resultados con los obtenidos en estudiantes de secundaria.

Se ha visto que los problemas combinatorios, tanto simples como compuestos, presentan un grado apreciable de dificultad para estos alumnos.

Se ha estudiado el efecto que, sobre la dificultad de los problemas, tienen diversas variables de tarea: esquema combinatorio, tipo de operación combinatoria y tamaño de la solución.

A partir del análisis de varianza se ha obtenido un efecto significativo de la operación combinatoria sobre la dificultad del problema y, además, se conserva el grado de dificultad de las operaciones respecto de estudios anteriores realizados con adolescentes. No se obtuvo, por el contrario, efecto significativo del esquema combinatorio sobre la dificultad del problema. La estimación del coeficiente de correlación lineal nos indicó la existencia de una relación inversa, moderada, entre el tamaño de la solución del problema y su índice de dificultad.

Se ha analizado la comprensión que los estudiantes muestran de los enunciados de los problemas.

La comprensión del enunciado no presenta especial dificultad, aunque se ha observado una cierta tendencia a convertirlo en uno de selección; los errores, en lo que se refiere al orden y la repetición, son escasos y suelen aparecer en los problemas de combinaciones y en los de permutaciones con repetición, respectivamente.

Se han analizado los métodos generales de resolución y las estrategias específicas utilizadas.

En lo que se refiere a los métodos generales hay que destacar un uso moderado de la enumeración y las fórmulas y, por el contrario, una ausencia casi total del diagrama de árbol. Técnicas basadas en la descomposición de problemas, fijación de variables y traducción a un problema equivalente son estrategias usadas con mayor o menor éxito en un alto porcentaje de problemas.

Se ha indagado sobre la influencia de nuevos factores explicativos, principalmente de tipo semiótico, sobre las estrategias, los errores y las dificultades en la resolución de problemas combinatorios simples y se ha iniciado el estudio de los problemas combinatorios compuestos.

La investigación muestra que, a pesar del carácter elemental de los problemas combinatorios seleccionados, los estudiantes tienen dificultades importantes para resolverlos debido a la estructura compleja de los procesos de resolución requeridos - puesta de manifiesto mediante un análisis de tipo semiótico- y a deficiencias en la enseñanza de la combinatoria, que enfatiza el estudio de las fórmulas de las operaciones combinatorias en detrimento de componentes más primarios del razonamiento combinatorio.

Se ha mostrado también que los componentes pragmáticos del razonamiento combinatorio no se desarrollan de manera espontánea en los sujetos a pesar, incluso, de la edad y una intensa preparación matemática y que, por tanto, requieren una atención especial en los procesos de instrucción matemática.

Los resultados obtenidos tienen un indudable interés por su fuerte implicación en la enseñanza de la combinatoria.

La escasa capacidad de razonamiento recursivo y de enumeración sistemática, muy generalizada en educación secundaria, la encontramos incluso en estudiantes de mayor edad y con una fuerte preparación matemática.

La combinatoria se ha manifestado como un campo en donde las estrategias de resolución de problemas, tales como dividir el problema en subproblemas, fijación de variables... pueden ser adecuadamente ejemplificadas y, por tanto, debe considerarse más un campo en el que aplicar y desarrollar herramientas heurísticas que el mero estudio de un tema cuya utilidad se restringe, la mayoría de las veces, a sus aplicaciones en el campo de la probabilidad.

\section{UN ESTUDIO DIDÁCTICO EN TORNO A LA ENSEÑANZA DE ASPECTOS BÁSICOS DE LA ELEC- TROSTÁTICA EN LA FORMACIÓN DE MAESTROS}

\section{Tesis doctoral}

Autora: Criado García-Legaz, Ana M. Director: Cañal de León, Pedro

Lugar: Departamento de Didáctica de las Ciencias. Universidad de Sevilla Programa: Didáctica de las Ciencias Experimentales

Fecha: 7 de febrero de 2000

La tesis se enmarca dentro de la línea de investigación sobre las concepciones de los estudiantes. También se hace una propuesta de unidad didáctica que se pone en práctica y se analiza su desarrollo y la evolución de las concepciones tras la misma. Pretende abordar el problema general: ¿Cómo mejorar la enseñanza y el aprendizaje de las nociones básicas de electrostática en alumnos de nivel universitario, con vistas a su formación como maestros?

Se presta atención al constructo de estatus cognitivo de las concepciones entendido como el compromiso cognitivo que un alumno mantiene con sus ideas, el cual se puede medir a través de indicadores como el grado de estructuración en sistemas de ideas, etc.

Tras un estudio teórico, de análisis de las dificultades y las posibles soluciones, se acotan los problemas a abordar:

1) ¿Cuáles son las concepciones de los alumnos del grupo estudiado sobre los conceptos básicos de electrostática, en cada momento de medición?

2) ¿Cuáles son sus características (grado de estructuración, estabilidad, dependencia del contexto, etc.) en cada momento de medición?

3) ¿Cómo evolucionan en relación con el desarrollo de una unidad didáctica?

Éstos, a su vez se desglosaron en subproblemas para concretar y fundamentar las hipótesis de la investigación.

El estudio se realizó con 52 estudiantes de la asignatura de Física General de magisterio. Como instrumentos se utilizaron un cuestionario y unas «hojas de respuesta» a las actividades de la unidad. Se utilizaron técnicas de análisis descriptivo, análisis bivariable y análisis multivariante; y fue administrado en tres etapas: test y retest antes de la enseñanza 
y postest, tras éste. El cuestionario permitió estudiar indicadores del estatus: la estabilidad sin mediar enseñanza, el grado de estructuración, la dependencia contextual y el índice de certeza.

\section{Resultados}

- Se analizó la identificación de fenómenos electrostáticos cotidianos y se describieron las concepciones sobre electrostática básica. Se identificaron y estudiaron las respuestas a cuestionarios sobre conceptos básicos de electrostática: los conceptos de cargado y de neutro; las interacciones entre objetos cargados: atracciones y repulsiones; la inducción entre un objeto cargado y otro neutro; y la posibilidad de electrizar metales por frotamiento.

- Sobre el grado de estructuración de las concepciones, se encontró una asociación, con significación estadística, en las ideas de los estudiantes. Algunas agrupaciones parecen poseer gran coherencia interna y otras son modelos híbridos con respecto a los formulados como hipótesis de trabajo. En cada momento de medición se detectaron y denominaron los modelos: de agitación de cargas; básico, de incremento de cargas en el materialy de reorganización de cargas dentro del material. Del test al retest, la pauta es de estabilidad pero con algunos cambios, reorganizándose las clases de alumnos dentro de los mismos modelos. En el postest, las respuestas se concentran en las modalidades correspondientes a concepciones de conocimiento académico en una tipología mayoritaria. Sólo permanecen ideas de un modelo híbrido entre los denominados reorganización de cargas y básico, asociado a una clase minoritaria. En el postest, las preguntas sobre cargado, repulsiones (en uno de los contextos), inducción y electrización de metales son las que marcan la diferencia entre las dos tipologías extremas de estudiantes. Persisten el modelo reorganización de cargas y la interpretación de la inducción desde el modelo básico (ambos objetos han de estar cargados), así como la idea de que los metales no se electrizan frotándolos con aislantes.

- Sobre la estabilidad test/retest y el cambio tras la enseñanza, se puso de manifiesto que: tomadas las respuestas una por una, las modalidades académicas no son necesariamente más estables que las concepciones alternativas como pauta general salvo en las atracciones entre objetos cargados. De entre las dos concepciones alternativas estables sobre la idea de cargado, la más estable es la de separación local de cargas. Ello puede deberse al peso de los conocimientos escolares previos en muchos estudian- tes. La baja estabilidad en las respuestas para las repulsiones y la inducción, comparada con los otros casos, debe responder al bajo estatus cognitivo de las interpretaciones sobre estos fenómenos. Sobre la electrización de los metales, la idea más estable es que los metales no se electrizan con aislantes, seguramente por ser derivada de otra reforzada en el contexto cotidiano: la diferencia de ambos en la conducción eléctrica.

- Sobre la dependencia contextual de las concepciones: tanto en el test como en el retest, sólo son independientes del contexto $\mathrm{y}$, por tanto, poseen mayor estatus cognitivo, las concepciones sobre las atracciones cargado-cargado y la inducción cargado-neutro, sean académicas o alternativas a ellas. Ambas tienen en común el hecho de que el fenómeno observado es una atracción, más familiar que las repulsiones, cuya interpretación cambia al cambiar de ejemplo. Además se halló una asociación entre el significado atribuido a cargado y a neutro cuando se trata de las ideas de agitación y las de reorganización pero no siempre, con los significados académicos. Por tanto aquellas concepciones, mantenidas independientemente del contexto serán mayores obstáculos que las interpretaciones usadas de forma menos sistemática. Tras la unidad didáctica, todas las respuestas académicas son significativamente independientes del contexto, si bien ello ocurre con menor frecuencia en la inducción y en la electrización de metales, lo cual redunda en los resultados anteriores.

- Sobre, el grado de certeza o plausibilidad, antes de la unidad didáctica, son más plausibles respuestas académicas para las preguntas sobre neutro, y las atracciones y repulsiones entre cuerpos cargados; y concepciones alternativas como: cargado significa polarizado, la inducción se produce por estar los objetos cargados con cargas de diferente signo y la idea de que los metales no se electrizan por frotamiento porque poseen los electrones unidos. Tras la unidad didáctica, las interpretaciones más plausibles son las respuestas académicas, pero también algunas concepciones alternativas sobre la inducción y las repulsiones.

- Concluyendo, se pueden establecer diferencias entre los conceptos según el estatus de las ideas utilizadas para interpretarlos: en un extremo, entrañan menos dificultad para los estudiantes analizados las atracciones entre cuerpos cargados y el concepto de neutro, pues existe un mayor estatus para las respuestas académicas. En el otro extremo están las interpretaciones de la inducción cargado-neutro y la electrización de metales para las cuales las concepciones alternativas poseen un alto estatus. En situación intermedia se encuentran las interpretaciones del significado de cargado y de las repulsiones.

LA ENSEÑANZA DE LA FÍSICA Y LA QUÍMICA EN LA EDUCACIÓN SECUNDARIA EN EL PRIMER TERCIO DEL SIGLO XX EN ESPAÑA

\section{Tesis doctoral}

Autor: López Martínez, José Damián Directores: Viñao Frago, Antonio; Bernal Martínez, J. y Mariano, $J$. Lugar: Departamento de Didáctica y Organización escolar. Universidad de Murcia Programa: La investigación y mejora de la calidad de la educación

Fecha: Noviembre de 1999

Este trabajo se enmarca en la línea de las investigaciones realizadas sobre la historia de las disciplinas y del currículo. El objeto fundamental es conocer los antecedentes, primeros pasos y evolución de la enseñanza de la física y la química en España en los niveles correspondientes a la educación secundaria. La investigación está estructurada en tres bloques.

1) En el primero se analizan los planes de estudio para la segunda enseñanza que han regido en España entre 1900 y 1936. Se estudia la situación de la física y la química respecto a otras áreas y al número total de horas de clase, así como la orientación que se proponía para su enseñanza. Para ello se han recopilado las disposiciones legislativas dictadas desde el Ministerio de Instrucción Pública así como otras propuestas realizadas que, a pesar de no entrar nunca en vigor, es necesario conocer. Se observa una evolución en los objetivos perseguidos con la enseñanza de la física y la química. El alumnado de los institutos durante el primer cuarto del siglo $\mathrm{xx}$ afrontaba por vez primera el estudio de la física y la química en los últimos cursos del bachillerato -si es que llegaban- con el único objetivo de servir de preparación para las carreras universitarias. Posteriormente se pasó al estudio de estas disciplinas de forma gradual y cíclica. No obstante, a lo largo del periodo estudiado, siempre fue menor el tiempo dedicado a la enseñanza de las ciencias experimentales que el programado para las materias propias del área de letras. A pesar del reconoci- 
miento, sobre el papel al menos, de la importancia que las ciencias físicoquímicas deberían tener en la educación de los ciudadanos, siempre se subestimó su valor formativo.

Al estudiar la evolución del currículo de estas disciplinas a través de algunos de los libros de texto más utilizados durante esos años y de los programas confeccionados por el profesorado, se consideran los cambios que se produjeron en las propuestas de selección y secuenciación de contenidos. En general, los libros de texto incluían un exagerado número de lecciones, con contenidos básicamente descriptivos sobre todas y cada una de las ramas de la física y de la química, sin actividades prácticas ni ejercicios o problemas para resolver por los alumnos y con un excesivo uso de los aparatos de demostración de leyes o principios físico-químicos para su corroboración. No obstante, de forma gradual se evoluciona desde textos con contenidos exclusivamente conceptuales -como listas de principios, leyes, propiedades, etc.-, puramente descriptivos y que debían ser aprendidos de forma memorística, a libros que incluyen unos contenidos más actualizados científicamente, que se van renovando, adoptando el estudio de aspectos fundamentales, con el planteamiento de experiencias prácticas, ejercicios y problemas que permitían el contacto personal del alumno con los fenómenos estudiados.

Mediante el análisis de las memorias anuales de los centros, de monografías publicadas sobre distintos institutos y de otras publicaciones de los propios profesores, se abordan también las condiciones en las que se desarrollaba la enseñanza de la física y la química en los institutos a través del material científico utilizado en los gabinetes y los laboratorios de los centros, las instalaciones de las que se disponía, el material que se adquiría y el que se proponía adquirir, qué experiencias prácticas de laboratorio se planteaban al alumnado y la evolución en dicho planteamiento en el período estudiado.

2) La segunda parte del trabajo surge al considerar que la historia de las disciplinas escolares es también la historia de quienes la enseñaron, del profesorado que impartía estas asignaturas. Conocer quiénes eran los integrantes del colectivo de catedráticos de física y química de instituto, su procedencia, su formación inicial, cómo se desarrollaban los procedimientos de acceso a las cátedras, cuál era su relación con las universidades, qué manuales, libros de texto y otras publicaciones de interés realizaron durante este período y su implicación en el contexto tecnológico, social, cultural y político de la época. Para ello hemos utilizado más de un centenar de expedientes personales de este colectivo de profesores y algunos de los expedientes de oposiciones a cátedras de instituto.

También consideramos los mecanismos que se arbitraron para propiciar su actualización científica y la formación pedagógico-didáctica tanto del profesorado en ejercicio como de los futuros catedráticos de física y química. En este sentido, hemos estudiado la labor realizada desde la Junta para Ampliación de Estudios a fin de establecer un sistema de becas que permitiera tanto la actualización científica como pedagógico-didáctica en centros dependientes de ella y en otros europeos. Asimismo, destacamos como desde el Instituto-Escuela de Madrid se llevó a cabo un modelo coherente de formación del futuro profesorado de ciencias de enseñanza secundaria.

3) Como tercer eje organizador de este trabajo se analizan las propuestas pedagógicas innovadoras que, en relación con la enseñanza de la física y la química, se hicieron durante el primer tercio del siglo xx en nuestro país. Se analizan las nuevas orientaciones didácticas y se profundiza en los aspectos relevantes que incidieron en la modernización y puesta en práctica de esas nuevas orientaciones metodológicas para la enseñanza de estas disciplinas. En este sentido, destaca la labor realizada en el Instituto-Escuela de Madrid por catedráticos del Instituto del prestigio de José Estalella, Andrés León, Miguel Catalán y los denominados aspirantes al magisterio secundario. La experiencia desarrollada en el I-E significó un cambio sustancial en los objetivos propuestos para la enseñanza de las ciencias en secundaria. Las nuevas orientaciones didácticas implicaban un cambio sustancial respecto al modelo de enseñanza tradicional. Se plantean los contenidos de la física y la química desde una nueva dimensión, a través de la familiarización de los alumnos con los hechos y los fenómenos de la vida cotidiana, mediante el planteamiento y resolución de problemas. Se reconoce el valor formativo de las salidas de campo y las visitas escolares. En lugar del tradicional libro de texto, se propone la utilización del cuaderno del alumno y los libros como material de consulta. Frente a las demostraciones y experimentos de cátedra, se recomienda la realización de actividades experimentales por parte de los alumnos, integradas en la secuencia de enseñanza, lo cual supondrá un cambio en el material científico utilizado.

Dentro de todo este movimiento de renovación de la enseñanza de las ciencias experimentales destaca con fuerza la figura de José Estalella, catedrático de física y química de Instituto. Sus ideas muestran un gran nivel de innovación didáctica: el alumno como protagonista esencial en el proceso de enseñanzaaprendizaje, la importancia otorgada a la ciencia de la vida cotidiana, el planteamiento y puesta en práctica de una alternativa al material científico y al trabajo práctico tradicional.

Por último, este trabajo refleja la repercusión que tuvieron esas nuevas orientaciones didácticas para la enseñanza de la física y química, recogidas en parte por la administración educativa (en el plan de estudios de 1934 y en otras propuestas realizadas bastantes años después). Al finalizar el primer tercio del siglo Xx, los institutos contaban ya con un grupo numeroso de profesores de física y química que gozaban de una excelente preparación científica y didáctica. Se daban las condiciones idóneas para desarrollar una enseñanza de las ciencias siguiendo principios similares a los puestos en práctica en el Instituto-Escuela de Madrid -o en sus análogos de Barcelona, Valencia o Sevilla. Se habría posibilitado una auténtica reforma de la enseñanza secundaria en nuestro país, pero la fractura que supuso la guerra civil y la posterior represión política -y también pedagógica-, truncó todo este proceso de renovación. 


\section{NOTICIAS}

LA CRISIS DE LA FÍSICA: UNA CRONICA DE LA SEMANA EUROPEA DE CIENCIA Y TECNOLOGÍA 2000

Mariano Merino de la Fuente

Departamento de Didáctica de las Ciencias Experimentales

Universidad de Valladolid

jema@dce.uva.es

\section{Introducción}

El pasado mes de noviembre de 2000 tuvo lugar en la sede del Laboratorio Europeo de Física de Partículas (CERN) un encuentro internacional auspiciado por el mencionado centro así como la Agencia Espacial Europea (ESA), el Observatorio Europeo Austral (ESO) y diversas sociedades nacionales de física, entre ellas, la Real Sociedad Española de Física (RSEF). En este evento intervinieron cerca de quinientos físicos europeos, tanto profesores como investigadores, con la común tarea de analizar las causas del declive del interés por los estudios de física (hecho comprobado en todos los países) y proponer remedios a esta situación.

Este magno evento tuvo caracteres de festival, feria y congreso. Este último aspecto se materializó en la celebración de varias mesas redondas que se ocuparon de debatir los aspectos más relevantes de la enseñanza de la física en todo el ámbito europeo. De esta forma, cerca de quinientos profesores de física de todo el continente tuvieron la oportunidad de cambiar impresiones, debatir problemas comunes y elaborar conclusiones.

La delegación española fue nutrida, en comparación a las de los restantes países. Estaba formada por 32 personas, de las cuales 10 eran directivos de la Rea Sociedad Española de Física, periodistas científicos y políticos vinculados al mundo de la educación; y 22 eran profesores de física, tanto de la universidad como de la etapa de secundaria. Estos últimos habían sido seleccionados mediante un concurso nacional celebrado ese mismo año.

Trece temas clave habían sido seleccionados para las distintas mesas redondas (http://www.cern.ch/pos). Los participantes se dispersaron en los diversos work- shops según sus preferencias y trabajaron durante tres días en el tema específico asignado. Transcurrido este plazo, los moderadores aportaron las conclusiones y recomendaciones, las cuales fueron presentadas y votadas por la Asamblea de Physics on Stage, formada por la totalidad de los participantes.

Se ha de señalar el papel activo de la delegación española, que estuvo presente en la práctica totalidad de los workshops; incluso ocupó el cargo de chairman en alguno de ellos.

A continuación, se reseñan los trece títulos de los debates con las cuestiones objeto de debate, un sumario del mismo y las recomendaciones finales. En todos los casos, hay una señalada con asterisco, que fue la más votada por la asamblea.

\section{Definición de la crisis}

\section{Cuestiones}

- ¿Hay crisis en la enseñanza de la física?

- ¿Disminuye el número de estudiantes que desean estudiar física?

- ¿Cuáles son las causas del problema? Sumario

Delegados de once países participaron en este debate, los cuales aportaron evidencias y datos de la existencia real de esta crisis en veintidós países. Entre las causas principales, se cuenta la estrecha banda de edades de los profesores de física, con un promedio de cincuenta años, combinado con un muy reducido influjo de los nuevos profesores (con una tasa de 5-10 profesores por millón de habitantes). Esta situación determinará una drástica reducción de los profesores de física en los próximos 5-10 años.

El número de estudiantes que eligen estudiar física, tanto en la etapa de secundaria como en universidad, decrece, si bien existe interés por la ciencia en el nivel de primaria.

Otros problemas discutidos fueron: la baja imagen de la física y la enseñanza en la sociedad, los efectos de los métodos de enseñanza actuales en los estudiantes, la exportación de estudiantes de calidad desde sus países de origen hacia Estados Unidos y la atracción de estudiantes de física por las altas remuneraciones de la industria, con menoscabo de su dedicación a la investigación científica.

\section{Recomendaciones}

- Introducir un sistema de recompensas para la formación del profesorado, sustentado por la industria y la comunidad científica, que valore y destaque las destrezas didácticas.

- Organizar un grupo de presión para mejorar la imagen de la física, el cual asegure que la escasez de medios económicos no afecte negativamente a los centros de enseñanza.

- Introducir la física en los medios de comunicación para concienciar a la sociedad y aumentar la conexión entre ésta y la vida ordinaria.*

- Incrementar la concienciación política sobre los problemas antes referidos.

- Reducir el aparato matemático en la enseñanza de la física, haciéndola más motivadora y agradable para la mayoría de los estudiantes.

- Concienciar a la sociedad de que las personas con formación en física son muy útiles en muchos campos de la actividad económica e industrial.

\section{La física en la educación primaria}

Cuestiones

- ¿A qué edad debe comenzar la educación científica?

- ¿Qué aspectos psicológicos inciden en las capacidades cognitivas en los primeros años?

- ¿Qué clase de física debe ser enseñada en esta etapa?: ¿conceptual o cuantitativa, práctica o teórica?

Sumario

Se identificaron diversos problemas relacionados con la educación para niños, tales como: 
- El escaso nivel de comprensión de los conocimientos de las ciencias en general y de la física en particular, por parte de los profesores.

- El nivel de comprensión del profesorado en torno al proceso de enseñanzaaprendizaje.

- Las actitudes del profesorado hacia la física, a menudo negativas y estereotipadas.

- Necesidad de que los profesores se hagan capaces de inculcar a sus alumnos formas científicas de pensar y trabajar.

- Comúnmente, la física se presenta descontextualizada a los niños.

- El conocimiento de la ciencia ha de preparar a los jóvenes para ser «adultos del futuro». Esta realidad debería ser reconocida y consensuada.

- Los profesores tienen que atender las necesidades de un alumnado diverso en capacidades y desarrollo. En consecuencia, no pueden basar únicamente sus métodos en el simple seguimiento de los textos escritos.

- Los profesores han de considerar las implicaciones de la física en el hogar, en el entorno local y en la vida ordinaria.

\section{Recomendaciones}

- Todos los niños de la Comunidad Europea deben tener acceso a experiencias relacionadas con la ciencia desde la más corta edad, esto es, desde su casa y el jardín de infancia hasta los doce años, y también después.

- Todos los profesores de primaria deberían tener acceso a la formación permanente en educación científica.*

- La Unión Europea debería poner a punto un «Centro virtual de recursos» accesible vía internet.

- Debería crearse una asociación europea de jóvenes científicos que potenciara la realización de actividades de los niños en la casa y la escuela.

- Todos los centros europeos de investigación deberían tener una división de educación.

\section{La física en la educación secundaria}

Cuestiones

- ¿Debe ser la física una materia obligatoria en el currículo escolar?
- La enseñanza preuniversitaria, ¿es para formar futuros científicos o para la educación general de los futuros ciudadanos?

- ¿Cuál es el nivel matemático aconsejable?

- ¿Debe ser el currículo de física más conceptual que cuantitativo?

- ¿Cómo puede relacionarse la física con otras materias?

Debido al elevado número de participantes, este workshop fue dividido en dos grupos: A (anglófono) y B (francófono).

Sumario del grupo A

El trabajo de este grupo se centró en las aportaciones de datos de los distintos países -a cargo de sus representantes-, el intercambio de experiencias y los requerimientos del currículo.

Se puso de manifiesto que la problemática de la enseñanza de la ciencia es muy parecida en todos los países. También se evidenció la gran variedad de criterios adoptados por los gobiernos y administraciones en sus respectivos sistemas educativos. En tercer lugar, se discutió largamente sobre la necesidad común de proporcionar una educación científica sólida, coherente con la relevancia que tiene y va a tener la física.

\section{Recomendaciones del grupo A}

- El aprendizaje de la ciencia en general y de la física en particular debe contextualizarse en la vida ordinaria.

- Los estudiantes deben aprender destrezas no sólo teóricas y matemáticas, sino también de información y comunicación tecnológica.

- Los métodos de enseñanza deben propiciar que los estudiantes lleguen a aprender por sí mismos.

- Los profesores deben reciclarse de forma continua.

- Los criterios gubernamentales para la enseñanza de la física deberían ser flexibles en el desarrollo del currículo.

- Debe financiarse el equipamiento de los centros y la formación, el reciclaje y la promoción del profesorado.*

\section{Sumario del grupo B}

Se destacó la necesidad de que existan grupos de discusión para estudiantes y profesores que debatan el estatus de la educación en física y se consideró muy conveniente que se traten todos estos temas en futuros eventos de esta misma naturaleza.

Por otro lado, se reconoció que la física es una parte importante de nuestra cultura actual y contribuye a una deseable amplitud de miras.

Se llegó también a la conclusión de que ser un buen profesor de física comporta no sólo conocer a fondo la materia sino también poseer destrezas en didáctica y pedagogía, y además, tener aptitudes y actitudes de investigador. Todo ello hace muy necesaria una buena formación didáctica.

\section{Recomendaciones del grupo $B$}

- Es preciso reconocer que la física es necesaria para casi todo, por ser parte de nuestra cultura.

- La enseñanza de la física debe ser desarrollada para todos los estudiantes, de los cuales la gran mayoría nunca serán científicos.

- La enseñanza de la física ha de ser realista y debe proporcionar explicaciones prácticas del mundo y de los acontecimientos que en él se dan.

- La enseñanza de la física ha de adaptarse a los diferentes niveles escolares y a los distintos intereses.

- Puesto que la física es una ciencia experimental, su enseñanza requiere recursos materiales y humanos adecuados, grupos de estudiantes de tamaño razonable y horarios adaptados.

- Los profesores de físicos han de tener conocimientos sólidos en física, didáctica y pedagogía y, además, actitudes aperturistas.*

\section{La física y la cultura científica}

\section{Cuestiones}

- ¿Pueden contribuir los programas de estudio actuales a la comprensión de la física?

- ¿Hasta qué punto contribuyen los programas de estudio actuales a la cultura científica?

- ¿Cómo puede presentarse la confusión entre ciencia y pseudociencia (cienciaficción)?

\section{Sumario}

La ciencia no es generalmente considerada como una parte importante de nues- 
tra cultura. Por el contrario, para el gran público, los términos física y química están cargados de connotaciones negativas, tales como: bomba atómica, plantas nucleares, polución ambiental, etc. La gente teme a la ciencia como si de un invento maligno se tratara y su motivación hacia ella es muy escasa, sobre todo debido a la forma en que se introduce en la escuela primaria y se trata en la secundaria. Se la considera al margen de los intereses normales. Incluso, el desconocimiento absoluto de la ciencia no es considerado como algo negativo, especialmente entre las mujeres.

Los científicos a menudo asumen que el público no comprende la ciencia; sin embargo, algunas personas acreditan tener conocimientos detallados de algunos aspectos de la ciencia, como, por ejemplo, la ingeniería del motor o la fabricación del vino.

No existen suficientes instituciones que se responsabilicen del conocimiento y comprensión pública de la ciencia. Se necesitan más museos interactivos y otros centros que promuevan su difusión pública, su enseñanza no reglada, una investigación encaminada hacia su comprensión, el apoyo a la escuela, la formación del profesorado, etc.

\section{Recomendaciones}

- Fomentar la realización de cursos de comunicación científica para periodistas, estudiantes de ciencias, científicos, etc.*

- Llevar la física a la gente mediante el establecimiento de redes de centros y otros programas de mayor alcance.

- Difundir la física por los medios de comunicación al más amplio sector del público, haciéndola accesible e interesante y relacionándola con otras disciplinas.

- Destacar los aspectos de la ciencia que respondan más y mejor a los intereses generales.

- Valorar la investigación en comunicación científica.

\section{Papel de la historia y la filosofía en la} enseñanza de la física

Cuestiones

- ¿Qué papel desempeña la historia de la ciencia en la enseñanza de la física?

- ¿Cómo puede la enseñanza de la física contribuir a la creencia de que la ciencia es buena o mala?

- ¿Qué responsabilidades morales son imputables a la enseñanza de la física?

\section{Sumario}

Una de las conclusiones a las que se llegó es que la historia de la ciencia se infrautiliza como herramienta pedagógica en la enseñanza de la física. En la mayoría de los casos, la escasa información de los profesores y la ausencia de interés de quienes diseñan el currículo determinan una muy escasa presencia de biografías de científicos y otros eventos históricos que, en el mejor de los casos, aparecen colateralmente o al final de las lecciones.

De hecho, es posible enseñar física sin ninguna referencia histórica, si bien una clara y profunda comprensión de los conceptos científicos pasa indefectiblemente por un buen conocimiento de su evolución histórica.

Se acordó que la historia de la ciencia y la filosofía que conlleva son elementos clave para la solución de los problemas que afectan a la enseñanza de la física.

\section{Recomendaciones}

- La historia y la filosofía de la ciencia deben formar parte de la formación de los profesores.*

- Debe facilitarse información acerca de los éxitos y fracasos del uso de la historia de la ciencia en la enseñanza.

- Las sociedades de historia y de ciencias deberían colaborar en estos empeños.

- Puede darse una imagen más realista de la ciencia a través de su historia y filosofía.

- El uso de la historia y filosofía de la ciencia clarifica las relaciones ente el mundo científico y otros campos del conocimiento.

Consideración de los principales temas de actualidad

\section{Cuestiones}

- Los temas de actualidad y la enseñanza de la física.

- ¿De qué forma podrían incluirse en la enseñanza de la física los temas actuales: bioingeniería, problemas medioambientales, desastres naturales, fuentes de energía, desarrollo sostenible, ciencia nuclear, etc.?

\section{Sumario}

Los participantes en este workshop acordaron que el tema principal es el problema del desarrollo sostenible. Todas las sociedades están afectadas por varios problemas de gran calado, y surgen cuestiones como, por ejemplo: ¿el consumo y la demanda de las futuras sociedades debe seguir el ritmo exponencial de crecimiento o bien debe tomar otros derroteros?
El equilibrio en el mundo es delicado y se ve fuertemente afectado por las actividades humanas. En estas condiciones, ¿cómo podemos mantener la línea de progreso de la humanidad? No hay otra alternativa que orientar a las futuras generaciones hacia la solución de estos problemas.

Los niños de hoy son los artífices del futuro a medio plazo y de la formación que les demos dependen las decisiones que ellos tomen. La física sustenta las nuevas tecnologías, varias de las cuales desempeñan un importante papel en la solución de los problemas relacionados con el desarrollo sostenible. No obstante, todos los delegados acordaron que la sociedad tiene un concepto negativo de la física por causa de acontecimientos de la historia reciente, desde la última guerra mundial, tales como la guerra fría, el incremento del arsenal nuclear y el ocultismo gubernamental que le es anejo, el accidente nuclear de Chernobil, etc.

\section{Recomendaciones}

- Es preciso reconocer que la física tiene aplicación inmediata en la comprensión y desarrollo de problemas relacionados con el crecimiento y el desarrollo sostenible.

- El currículo debería dirigirse hacia los temas señalados arriba y hacia el impacto de la física en la vida ordinaria.*

- El ritmo de la investigación y el incremento de la información reclaman una buena formación de los profesores.

- Es imprescindible la cooperación internacional a la hora de diseñar y desarrollar los currículos, en el ámbito local y nacional.

- Debe proporcionarse tanto a profesores como a estudiantes una información fiable, la cual está disponible y libre en la propaganda.

- El currículo debe permitir que los estudiantes tomen decisiones razonadas, sopesando pros y contras en un contexto económico y social.

\section{La mujer y la física}

\section{Cuestiones}

- ¿Qué es la estadística? (contribuciones nacionales)

- ¿Qué debe hacerse para igualar los desequilibrios por razón de sexo en ciertos países?

- ¿Por qué las mujeres prefieren otras ciencias (p.e., biología) antes que la física? 


\begin{abstract}
Sumario
Las discusiones de este grupo se centraron fundamentalmente en dos bipolaridades -hombres/mujeres y chicos/chicasy se aportaron datos estadísticos, básicamente de la Unión Europea y del American Institute of Physics.

Hay una gran diferencia de proporción entre hombres y mujeres que aparecen en las distintas carreras profesionales. Existen muy pocas mujeres en la cúpula de la jerarquía académica, no sólo en física, sino en todas las ciencias. Incluso en e caso de las ciencias «blandas», los hombres dominan los puestos más altos del escalafón.
\end{abstract}

Se constató que existe en varios países una intensa y subliminal discriminación hacia las mujeres y se instó a que hombres y mujeres sean conscientes de este peligro oculto.

Se discutió sobre la conducta de los chicos y las chicas en la escuela. Se puso de evidencia que las muchachas carecen a menudo de la suficiente confianza en sí mismas, tienden a pensar que su trabajo no es importante y que la física no es para ellas. Los profesores deberían ser sensibles a este hecho y tenerlo en cuenta cuando enseñan física. Como remedio posible, deberían enfatizar en los aspectos culturales, históricos, humanísticos, médicos, etc., de la física, que resultan más atractivos para ellas.

\section{Recomendaciones}

- El conocimiento de la problemática del sexo y la ciencia debería formar parte de la formación del profesorado.*

- Diseñar y desarrollar planes de ayuda a la mujer con hijos pequeños: jardines de infancia, horarios de trabajo fraccionados, etc.

\section{Física y juego}

\section{Cuestiones}

- ¿Qué papel desempeña el juego en la enseñanza de la física?

- ¿Qué clase de juegos y juguetes ilustran los principios físicos importantes?

- Comparación de diversas experiencias.

\section{Sumario}

Los componentes de este grupo, tras las oportunas aportaciones y debates, llegaron a una serie de conclusiones concretas:
El juego y los juguetes son un excelente medio de iniciación, con ellos pueden introducirse nuevos conceptos. Los juegos que plantean cuestiones son los mejores, estimulan la imaginación, captan poderosamente la atención y suscitan la discusión.

Los juegos y los juguetes traspasan las barreras del lenguaje, evidencian que la física está presente en nuestro entorno, son esenciales para iniciarse en la física en la escuela primaria, ayudan a la gente a comprender la tecnología y, además, son baratos de adquirir.

Finalmente, los juegos y los juguetes son intrínsecamente motivadores, dan la sensación de que cualquiera puede hacer física; en cierto modo, convierten al profesor en un alumno más. No se limitan a proporcionar una satisfacción efímera sino que proporcionan un gozo duradero y fructífero.

\section{Recomendaciones}

- Concienciar a los profesores de que el juego y los juguetes tienen una función altamente motivadora: provocan cuestiones y estimulan la actividad investigadora.*

- Animar a los profesores a que jueguen con sus alumnos y hagan del aprendizaje una diversión.

- El juego y la comprensión científica debería formar parte de la formación del profesorado.

- Incrementar la convicción de que el juego permite traspasar la barrera del lenguaje.

\section{El lugar de internet en la enseñanza de} la física

Cuestiones

- ¿Cómo puede beneficiarse la escuela de la información disponible en internet?

- ¿Cómo puede utilizarse internet para promover la comunicación?

- ¿Hay facilidades para ello?

\section{Sumario}

En los últimos diez años, el desarrollo y crecimiento de la llamada sociedad de la información ha cambiado nuestra forma de vivir. Nuevas herramientas afectan a todos los órdenes de nuestra actividad y, de forma particular, a la educación.

Es indudable el poder de internet, si bien ello no es suficiente: tenemos ante nosotros una herramienta y ahora tenemos que aprender a utilizarla en educación. Es preciso organizar el gran bagaje de información y coordinar a los diferentes grupos que trabajan por la consecución de las mismas metas. Se trata, sin duda, de uno de los mayores retos actuales.

En este workschop se discutieron estos problemas y se intercambiaron experiencias sobre lo que se hace en los distintos países. La falta de una organización que coordine los esfuerzos de los distintos grupos se configura como el mayor problema, así como la financiación de dicha organización.

Quedó claro que la coordinación, la cooperación y la participación en las diversas experiencias son las claves fundamentales de la introducción de internet en la educación.

\section{Recomendaciones}

- Globalizar la red europea de educación.

- Garantizar la existencia y financiación de futuros sitios.

- Desarrollar una investigación de calidad por y para los profesores, sobre el proceso de aprendizaje basado en el empleo de internet.

- Investigar, desarrollar y evaluar métodos para el uso de internet en la enseñanza.*

- Llevar la enseñanza basada en internet a los programas de desarrollo profesional en cada país.

\section{Nuevas herramientas en la clase}

\section{Cuestiones}

- ¿Cómo puede potenciarse el empleo de las nuevas tecnologías en la enseñanza de la física?

- ¿Qué beneficios para la enseñanza se derivan del empleo de ordenadores y $\mathrm{CD}$ ?

- ¿Cómo pueden usarse para la realización de proyectos y demostraciones?

\section{Sumario}

La física es excitante no sólo para los físicos sino también para los jóvenes, y debemos esforzarnos en proporcionar medios de motivar a la población joven para que disfrute con ella. En este sentido, las nuevas tecnologías proporcionan oportunidades inigualables para ello, por ejemplo, facilitando la compartición de ideas y la información entre sectores de población cada vez mayores. 
Las variaciones en el uso de las nuevas tecnologías en la enseñanza de la física reflejan la diferencias que existen en el conjunto de la sociedad. Quedó claro para los miembros de este workshop que hay grandes diferencias entre la provisión de nuevas tecnologías y la necesaria especialización requerida para su uso. Si la enseñanza de la física contribuye a la preparación de los jóvenes para un mundo cambiante y tecnificado, es obligado incorporar a ésta las nuevas tecnologías.

\section{Recomendaciones}

- Es preciso financiar la introducción de las nuevas tecnologías en la escuela.

- El soporte financiero para la formación del profesorado en temas relativos a la tecnología de la información y la comunicación (ICT) debería atender a los aspectos pedagógicos.*

- Es necesaria la creación de una central de datos que permita el intercambio de información en el uso de ICT en la enseñanza de la física.

- La enseñanza de la física debe ser reflejo de la sociedad y usar las ICT, revalorizando y extendiendo la experiencia del aprendizaje.

- Deberían crearse asociaciones entre los centros de enseñanza de los distintos niveles y la industria para fomentar el acceso de los estudiantes a las nuevas tecnologías.

- Es preciso desarrollar la compatibilidad entre los diferentes equipamientos ICT.

Los grandes organismos científicos y la enseñanza de la física

\section{Cuestiones}

- ¿Qué contribución pueden y deben hacer las grandes organizaciones científicas a la enseñanza de la física?

- ¿A qué niveles debe hacerse esta contribución?

- ¿Qué propósitos a largo plazo hay en este sentido?

\section{Sumario}

Las organizaciones científicas europeas han comenzado ya actividades tendentes a estimular la concienciación y el interés por las ciencias de la naturaleza.

Delegados de once países participaron en este workshop con representantes de
CERN, ESA, ESO y UE y debatieron acerca del desarrollo de los proyectos en curso, si bien el mayor peso del debate se centró en la educación como un asunto clave para el futuro de Europa.

Se discutió sobre la diseminación del conocimiento a través de las redes existentes y su mejoramiento a través de una coordinación sinérgica entre las organizaciones científicas europeas y nacionales.

También fueron objeto de atención las formas de involucrar a los profesores en programas educativos europeos e incrementar su interacción con las organizaciones científicas.

En cuanto a los asuntos de la financiación, se señaló que los programas educacionales desarrollados por las sociedades científicas deberían ser complementados con aportaciones y patrocinio de la Comisión Europea (Sexto Programa Marco y posiblemente otros programas EC).

\section{Recomendaciones}

- La educación es un tema prioritario para Europa y las organizaciones científicas europeas deben involucrarse en él.

- La educación y el desarrollo deben ser integrados con los proyectos de investigación, destinando un $1 \%$ de su presupuesto.*

- La Unión Europea, las organizaciones europeas de física (European Physical Society, European Association for Astronomy Education, etc.), las asociaciones de profesores y las organizaciones nacionales deben promover actividades, tanto a corto como a largo plazo.

- Las organizaciones europeas de física (EPS, EAAE, etc.) deberían disponer de profesores residentes en comisión de servicios que llevaran adelante actividades con soporte de programas EC.

- Deberían adaptarse las bases de datos de las organizaciones europeas de física para fines educativos.

\section{El oficio de profesor}

Cuestiones

- ¿Cómo está el mercado del trabajo para los profesores de física?

- ¿Es aceptable el estatus social del profesor?

- ¿Se precisa más colaboración en Europa en el campo de la enseñanza de la física?

\section{Sumario}

Este grupo de trabajo se centró en los aspectos y circunstancias que afectan a la captación de nuevos profesores y cómo éstos pueden modificarse para superar la crisis que afecta a la enseñanza de la física. En algunos países, la escasez de profesores de física es verdaderamente crítica y la preparación de los pocos que acceden a la profesión es pobre. Por el contrario, en otros países no hay escasez de profesores si bien la preparación pedagógica que acreditan los aspirantes es en general baja.

\section{Recomendaciones}

- Es preciso mejorar las condiciones de trabajo de los profesores (horarios de clase, horas de preparación, ayudas técnicas, formación permanente, desarrollo profesional, perspectivas de promoción etc.).*

- Los salarios de los profesores deben ser equiparados a los de otros profesionales de igual nivel de cualificación.

- Los profesores requieren una buena formación inicial y un posterior desarrollo profesional. Al igual que en otras profesiones, se debería recompensar económicamente la cualificación profesional.

- Se recomienda la promoción de los contactos personales entre los profesores de diferentes países en orden al intercambio de experiencias educativas.

- Sería necesario llevar a cabo un proceso de auditoría de calidad, cantidad y competencias de los profesores en toda Europa.

- En la mayoría de los países está decreciendo el respeto y la consideración hacia el profesorado por parte de alumnos, padres y sociedad en general. Esta tendencia puede ser modificada favorablemente desde los gobiernos y administraciones.

\section{Desarrollo del currículo}

\section{Cuestiones}

- Comparación de nuevos proyectos curriculares.

- Cambio de ideas: participación y adaptación.

- ¿Qué tipo de organización se necesitaría para asegurar que las buenas ideas fueran ampliamente puestas en práctica?

\section{Sumario}

Con respecto a la complejidad del currículo en Europa, este workshop se con- 
centró en un número de publicaciones que parecen ser las más importantes en la enseñanza de la física en todos los países y que pueden ser las que más y mejor influyan en el desarrollo curricular. Básicamente, las discusiones se centraron en los siguientes aspectos:

- ¿Cuales son las finalidades del currículo?

- ¿Puede el currículo dirigir la ciencia hacia todos o solamente a los especialistas?

- ¿Qué autonomía puede tener el profesor y cuánta el alumno?

- El currículo, ¿ha de tener sólo contenidos o también ha de describir contextos?

- ¿Cómo son las conexiones entre currículo y evaluación?

- ¿Cómo puede asegurarse el progreso en la educación?

- ¿Cómo pueden reflejarse las innovaciones en el currículo?

\section{Recomendaciones}

- Es preciso que los currículos estén bien definidos: objetivos, contenidos, contexto, metodología, grado de flexibilidad y resultados esperados.

- El proceso de desarrollo de un currículo debería incluir los siguientes pasos: innovación, participación, realización y evaluación.

- Un currículo debe ser algo vivo, sujeto a continuas mejoras, resultado de la evaluación a partir de la participación de todos los estamentos involucrados en él.*

\section{NOTA}

Pueden obtenerse más detalles acerca de cada workshop así como explicaciones sobre las recomendaciones en la web: http://www.cern.ch/ pos o bien solicitándolo a:

Helen Wilson.

Executive Coordinator for Physics on Stage ESTEC (ADM-RE)

European Space Agency

Keplerlaan 1

2200 AG Noordwijk

The Nederlands $2^{\text {nd }}$ INTERNATIONAL CONFERENCE ON THE TEACHING OF MATHEMATICS

Fecha y lugar: Del 1 al 6 de julio de 2002 en Creta. Grecia.

Inscripción e información:

http://www.math.uoc.gr/ ictm2

XVI REUNIÓN LATINOAMERICANA DE MATEMÁTICA EDUCATIVA

Fecha y lugar: Del 15 al 19 de julio de 2002 en La Habana. Cuba.

Inscripción e información:

http://www.clame.org.mx/relme16/ index.htm

\section{CIEAEM 54}

Fecha y lugar: Del 13 al 19 de julio de 2002 en Vilanova y La Geltrú. Cataluña. España.

Inscripción e información:

Joaquín Giménez, Facultad de Ciencias de la Educación. Universidad de Barcelona. España.

jgimenez@campus.uoc.es

\section{PME 26}

Fecha y lugar: Del 21 al 26 de julio de 2002 en la Universidad de East Anglia. Norwich. Reino Unido.

Inscripción e información: Anne Cockburn, School of Education \& Professional Development, Norwich NR4 7TJ, Reino Unido.

Tel: +44 - 1603592644

Fax: +44 - 1603593446

pme26.chair@uea.ac.uk

http://www.uea.ac.uk/edu/pme26

\section{ERME 2002, SUMMER SCHOOL}

Fecha y lugar: del 23 al 27 de agosto de 2002 en Klagenfurt. Austria.
Inscripción e información:

http://yerme2002.uni-klu.ac.at/

XX ENCUENTROS DE DIDÁCTICA DE LAS CIENCIAS EXPERIMENTALES

Fecha y lugar: Del 11 al 13 de septiembre de 2002 en La Laguna. Tenerife, España.

Organiza: Asociación Española de Profesores e Investigadores en Didáctica de las Ciencias Experimentales (APICE)

Inscripción e información:

Secretaría de los «XX Encuentros de Didáctica de las Ciencias Experimentales». Instituto de Ciencias de la Educación. Centro Superior de Educación. C/ Heraclio Sánchez 37. Universidad de La Laguna. 38204 La Laguna. Tenerife.

Tel: 34922319035

Fax: 34922319037

inscripcion.apice@ull.es

http:// webpages.ull.es/users/apice/

\section{SIMPOSIO DE LA SEIEM}

Fecha y lugar: del 11 al 14 de septiembre de 2002, la Universidad de La Rioja, Logroño, España.

Inscripción e información:

Jesús Murillo Ramón. Universidad de La Rioja. Departamento de Matemáticas y Computación. Edificio VIVES. Universidad de La Rioja. C/ Luis de Ulloa s/n. 26004. Logroño.

Tel.: 941.299452.

Fax.: 941299460

http://www.ugr.es/local/seiem/

THE HUMANISTIC RENAISSANCE IN MATHEMATICS EDUCATION

Fecha y lugar: Del 20 al 25 de septiembre de 2002 en Palermo. Sicilia. Italia.

Inscripción e información:

Alan Rogerson

arogerson@vsg.edu.au 